\title{
Hyperoxia downregulates angiotensin-converting enzyme-2 in human fetal lung fibroblasts
}

\author{
Chinyere I. Oarhe' ${ }^{1}$, Vinh Dang ${ }^{2}$, MyTrang Dang ${ }^{3}$, Hang Nguyen ${ }^{4}$, Indiwari Gopallawa ${ }^{4}$, Ira H. Gewolb ${ }^{1}$ and Bruce D. Uhal ${ }^{2}$
}

\begin{abstract}
BACKGROUND: Angiotensin (ANG) II is involved in experimental hyperoxia-induced lung fibrosis. Angiotensin-converting enzyme-2 (ACE-2) degrades ANG II and is thus protective, but is downregulated in adult human and experimental lung fibrosis. Hyperoxia is a known cause of chronic fibrotic lung disease in neonates, but the role of ACE-2 in neonatal lung fibrosis is unknown. We hypothesized that ACE-2 in human fetal lung cells might be downregulated by hyperoxic gas.
\end{abstract}

METHODS: Fetal human lung fibroblast IMR90 cells were exposed to hyperoxic $\left(95 \% \mathrm{O}_{2} / 5 \% \mathrm{CO}_{2}\right)$ or normoxic $(21 \%$ $\mathrm{O}_{2} / 5 \% \mathrm{CO}_{2}$ ) gas in vitro. Cells and culture media were recovered separately for assays of ACE-2 enzymatic activity, mRNA, and immunoreactive protein.

RESULTS: Hyperoxia decreased ACE-2 immunoreactive protein and enzyme activity in IMR90 cells (both $P<0.01$ ), but did not change ACE-2 mRNA. ACE-2 protein was increased in the cell supernatant, suggesting protease-mediated ectodomain shedding. TAPI-2, an inhibitor of TNF- $\alpha$-converting enzyme (TACE/ADAM17), prevented both the decrease in cellular ACE-2 and the increase in soluble ACE-2 (both $P<0.05$ ).

CONCLUSION: These data show that ACE-2 is expressed in fetal human lung fibroblasts but is significantly decreased by hyperoxic gas. They also suggest that hyperoxia decreases ACE-2 through a shedding mechanism mediated by ADAM17/ TACE.

$S_{n}^{n}$ upplemental oxygen, which is frequently used in the treatment of pulmonary insufficiency in premature infants, has been implicated in the development of bronchopulmonary dysplasia (BPD). BPD remains a major cause of morbidity and mortality during the first year of life, and some of these infants have significant respiratory problems throughout childhood, including increased airway reactivity and the development of restrictive airway disease (1). Hyperoxia induces lung injury and may contribute to BPD. A comprehensive review of the literature reveals a strong association between exposure to hyperoxia and subsequent expression of comorbidities. Hyperoxia can directly cause lung injury by generation of reactive oxygen radicals. Prolonged exposure of neonatal mice to hyperoxia results in impaired alveolarization and capillary development and in increased lung fibrosis that is similar to human BPD (2).

Among the mechanisms believed to contribute to the pathogenesis of lung fibrosis, ACE-2 is of great importance, since the renin-angiotensin system (RAS) is expressed by and is functional in human lung myofibroblasts, the cells believed to be most important in synthesizing the collagens that accumulate in fibrotic lungs (3). In studies of tissue fibrogenesis, the tissue RAS is believed to play a key role in initiation and progression of fibrous tissue accumulation in a variety of organs. Both ACE inhibitors and Angiotensin receptor antagonists have been shown to prevent fibrogenesis of the heart (4), liver (5), kidney (6), and lungs $(7,8)$. ANG II, produced by ACE, is the main effector molecule in the RAS. ANG II is a potential pulmonary profibrotic mediator which activates ANG II type I receptor and stimulates collagen synthesis. Studies done in vivo showed that the angiotensin system was upregulated in oxygen-exposed fetal lung fibroblasts (9). The main function of ACE-2 is to convert the octapeptide angiotensin II to a heptapeptide ANG 1-7. This limits the accumulation of ANG II and protects against experimental lung fibrosis.

Imai et al (10). demonstrated in three different models of acute respiratory distress syndrome (ARDS) which all used ACE 2 knock-out mice, that these mice showed very severe disease compared with wild-type mice. Previous work from this laboratory demonstrated that ACE-2 protects against fibrosis, but is downregulated in both human and experimental lung fibrosis models (11), and that inhibition of ACE-2 is associated with increased lung tissue injury/fibrosis. Earlier, Uhal et al (3). demonstrated the existence of an angiotensin/ TGF- $\beta 1$ "autocrine loop" in human lung myofibroblasts. More important in the context of the neonatal lung, Jiang et al (12). showed the activation of the angiotensin system in hyperoxiainduced lung fibrosis in neonatal rats. Chou et al (13). showed that blocking ANG II activity through the AT1R (angiotensin type I receptor) blocker losartan prevented lung fibrosis in response to hyperoxia. The role of ACE- 2 in these models, however, has not yet been evaluated.

The function of ACE-2 as the cellular receptor for SARSCoronavirus was demonstrated by Li et al (14).. Haga et al (15). 
demonstrated the role of TNF- $\alpha$-converting enzyme (TACE), which can cleave the ectodomain of ACE-2, in viral entry into the lungs. These authors also showed that TACE-dependent "shedding" of ACE-2 ectodomain is completely dependent on the binding of SARS-S protein to ACE-2 and that TACE was required for viral entry (16). Further, the shedding was blocked by TACE antagonists, TAPI-0, and TAPI-2 (15). TACE (also known as ADAM17), originally identified as a metalloprotease required for processing the membrane form of TNF$\alpha$ and its release as a soluble factor (17), is now known to be involved in the cleavage and release of the ACE-2 ectodomain in several tissues (18).

Given the known involvement of other RAS components in lung fibroblast function (3) and the profibrotic action of hyperoxic gas, we hypothesized that ACE- 2 might be expressed by fetal lung fibroblasts and further, might be downregulated by hyperoxia. We further theorized that the downregulation of ACE-2 by hyperoxia might be prevented by inhibiting its shedding by an inhibitor of TACE, the enzyme that causes shedding. We report here the findings that ACE-2 protein and enzyme activity are severely decreased by hyperoxia in human fetal lung fibroblast cultures. We also report that TAPI-2 prevents this decrease.

\section{RESULTS}

\section{Cell Viability Data}

Table 1 shows the number of cells with disrupted plasma membrane integrity, as measured by trypan blue and expressed as a percentage of total cells. Cell cultures exposed to hyperoxia showed a modest (less than 1\%) but statistically significant increase in dead cells compared to cells exposed to room air, if the exposure was followed by a 24-h period of recovery $(P<$ $0.01)$. Figure 1 shows western blotting for caspase 9 as a marker of apoptosis which, when normalized to $\beta$-actin, showed no significant changes by densitometry. Together, these data suggest a very slight decrease in the viability of hyperoxia-treated cells following a recovery period, but only in a very minor fraction $(<1 \%)$ of the cells.

\section{Effect of Hyperoxia on ACE-2}

Figure 2 shows the effect of hyperoxia on ACE-2 immunoreactive protein on western blotting. Both ACE- 2 immunoreactivity and enzyme activity were significantly decreased in cells treated with hyperoxia when compared with cells treated with normoxia. This reduction $(74.6 \%, P<0.01$ by densitometry) only occurred when cells were allowed to recover by incubating in normoxic serum free media for $24 \mathrm{~h}$.

Table 1. Loss of plasma membrane integrity assessed by Trypan Blue

\begin{tabular}{lcccc}
\hline & $\begin{array}{c}\text { Control with } \\
\text { no recovery }\end{array}$ & $\begin{array}{c}\text { Hyperoxia with } \\
\text { no recovery }\end{array}$ & $\begin{array}{c}\text { Control with } \\
\text { recovery }\end{array}$ & $\begin{array}{c}\text { Hyperoxia } \\
\text { with recovery }\end{array}$ \\
\hline Mean (\%) & 0.08 & 0.10 & $0.47^{*}$ & $0.7^{* * *}$ \\
SD & 0.011 & 0.07 & 0.03 & 0.09 \\
\hline
\end{tabular}

IMR90 cells were exposed to hyperoxic or normoxic gas as described in Materials and Methods. Immediately thereafter, trypan blue was added directly to the cell culture wells for determination of cell death by loss of dye exclusion. Values are the mean of at least three determinations each; ${ }^{*} P<0.001$ vs. control no recovery and ${ }^{* *} P<0.01$ vs. corresponding Control by ANOVA and Tukey-Kramer multiple comparisons test.

\section{Confirmation of ACE-2 Expression With a Competitive} Inhibitor, DX 600

In Figure 3, the enzymatic activity measured by the ACE-2 synthetic substrate was completely inhibited by the synthetic peptide $\mathrm{DX}_{600}(P<0.01)$, a competitive inhibitor of ACE-2 (11). Figure $3 \mathbf{b}$ showed no significant reduction of ACE-2

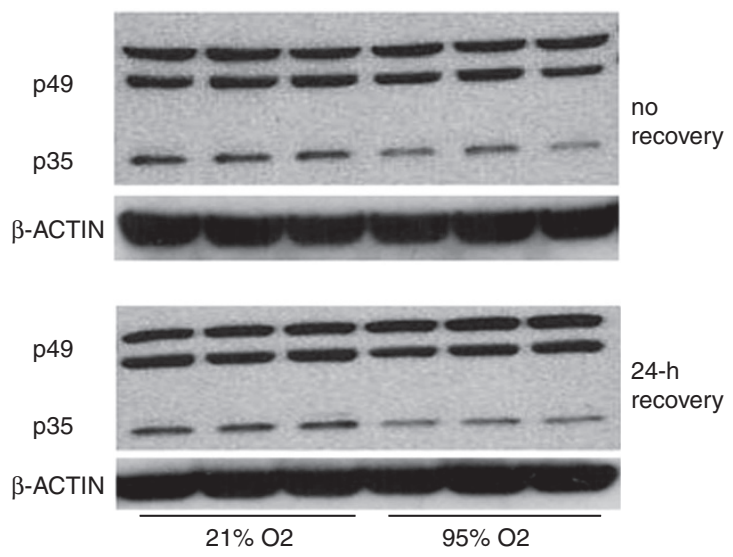

Figure 1. Caspase 9 in fetal lung fibroblasts exposed to hyperoxic or normoxic gas. IMR 90 cells in confluent culture were exposed for $72 \mathrm{~h}$ to $95 \%$ oxygen with $5 \% \mathrm{CO}_{2}$. Control cells were incubated in $21 \% \mathrm{O}_{2}$ with $5 \% \mathrm{CO}_{2}$ for $72 \mathrm{~h}$. At the end of $72 \mathrm{~h}$, cells were either harvested or incubated for an additional "recovery" phase in serum free media for $24 \mathrm{~h}$. Western blotting for caspase 9 was then performed as described in Materials and Methods. Despite the slight decrease in band intensity in hyperoxia-treated cells, quantitation by densitometry demonstrated no statistically significant difference in the caspase $9 / \beta$-actin ratio (not shown).

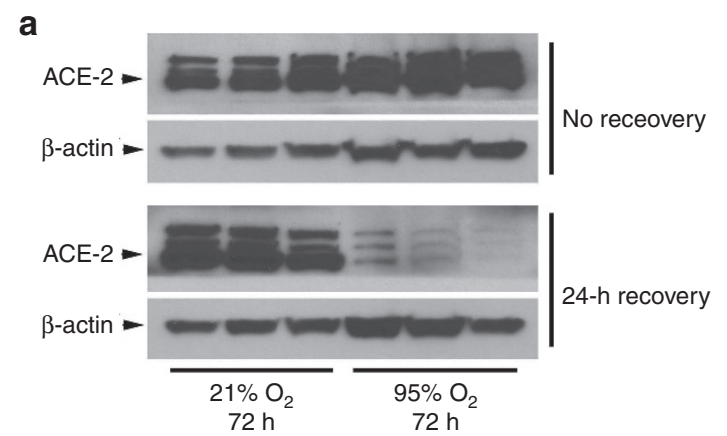

b

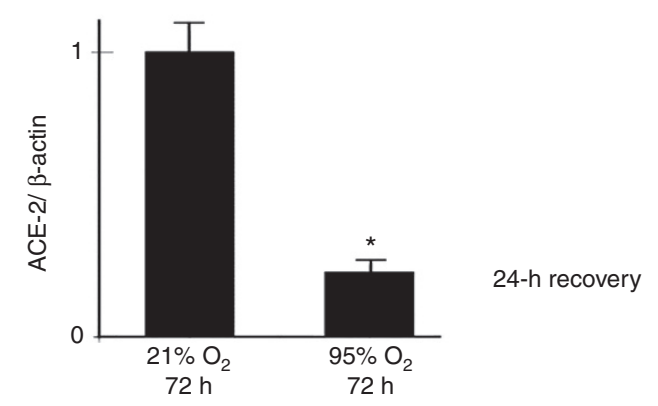

Figure 2. Hyperoxia with normoxic recovery downregulates angiotensinconverting enzyme-2 (ACE-2) in human fetal lung fibroblasts. IMR90 cells were exposed to hyperoxic or normoxic gas as described in Figure 1. (a) Western blotting for ACE-2 protein was then performed and normalized to $\beta$-actin. (b) There was no change in ACE- 2 without recovery but with recovery hyperoxia caused a significant decrease in ACE-2 immunoreactive protein $\left(74.6 \%,{ }^{*} P<0.01\right.$ vs. $21 \% \mathrm{O}_{2}$ by Student's $t$-test). 
mRNA by hyperoxia, suggesting that reduction of ACE-2 is not occurring at the RNA level but at the protein level.

\section{Effect of TAPI-2 on ACE-2 in Fetal Lung Cells Exposed to Hyperoxia}

Figure 4 shows that the addition of TAPI-2 during exposure to hyperoxia prevented the reduction of immunoreactive ACE-2 that otherwise would occur in hyperoxia-treated cells $(P<0.05)$
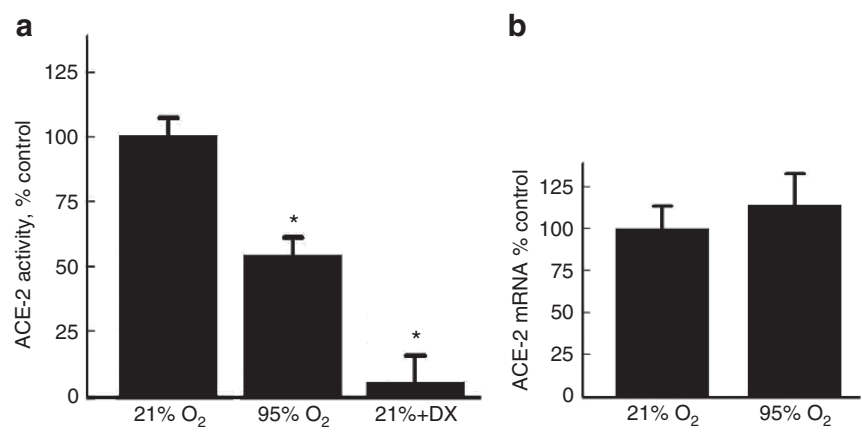

Figure 3. Hyperoxia with normoxic recovery reduces angiotensin-converting enzyme-2 (ACE-2) enzymatic activity but not ACE-2 mRNA. IMR90 cells were exposed to hyperoxic or normoxic gas as described in Figure 1 (with recovery only), but cells were harvested for assay of ACE-2 enzyme activity (11) with and without inclusion of the ACE-2 competitive inhibitor DX600 at $1 \mu \mathrm{mol} / \mathrm{l}$ in the enzyme assay vessel. (a) ACE- 2 enzymatic activity showed a statistically significant reduction by hyperoxia (with recovery) and complete blockage by $\mathrm{DX}_{600}\left({ }^{*} P<0.01\right.$ vs. $21 \% \mathrm{O}_{2}$ by ANOVA and Dunnett's test). The (b) ACE-2 mRNA quantification by qRTPCR showed no significant change. See Methods for details.
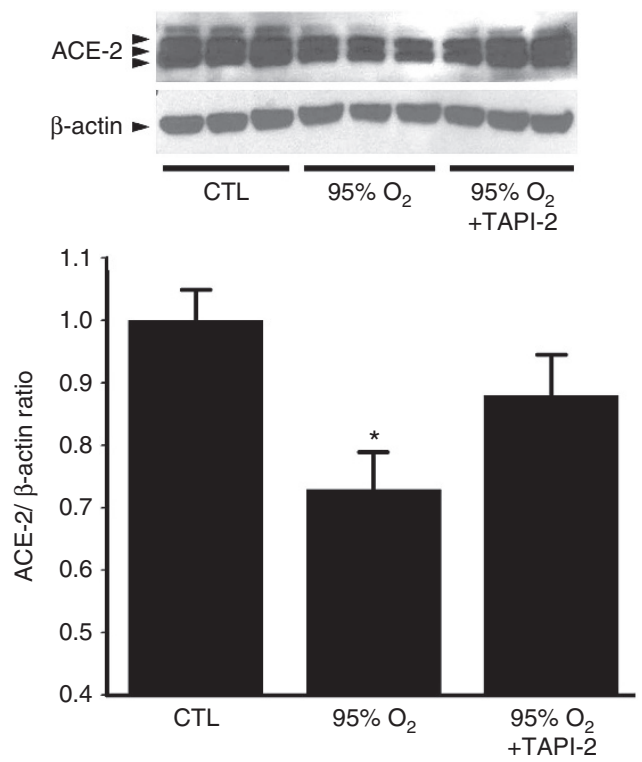

Figure 4. Hyperoxia with normoxic recovery reduces cellular angiotensinconverting enzyme-2 (ACE-2) by a TAPI-2-sensitive mechanism. IMR90 cells were exposed to hyperoxic or normoxic gas as described in Figure 1 (with recovery), but in the presence or absence of the ADAM17/TACE inhibitor TAPI-2. Cell monolayers were then harvested for ACE-2 western blotting (upper panel). Densitometry (lower panel) demonstrated a significant reduction in cell monolayer-associated ACE- 2 in hyperoxia treated cells compared to the control ( ${ }^{*} P<0.05$ vs. CTL by ANOVA and Tukey's multiple comparison test). Addition of TAPI-2 prevented the decrease.
Effect of TAPI-2 on Soluble ACE-2 in Cell-Free Culture Supernatants

Next, ACE-2 immunoreactivity was analyzed in the culture media collected from hyperoxia-exposed cells. Figure 5 shows that treatment of fetal lung fibroblasts with hyperoxia increased the release of soluble ACE- 2 into the culture supernatant $(P<$ $0.01)$ and that TAPI-2 prevented the increase $(P<0.01)$.

\section{Effect of Hyperoxia With Normoxic Recovery on ADAM17/TACE}

Figure 6 shows that the treatment of hyperoxia followed by normoxic recovery significantly increased ADAM17/TACE immunoreactive protein by threefold in the IMR90 cells $(P<0.05)$. The hyperoxic gas alone (without recovery) did not increase ADAM17/TACE (data not shown). As expected, the inclusion of the TACE inhibitor TAPI-2 (an active site blocker) had no ability to reduce the increase in TACE protein induced by hyperoxia with normoxic recovery (data not shown). Figure 6, panel c shows that hyperoxia with normoxic recovery also caused a significant increase in ADAM17/TACE mRNA (2.2-fold, $\left.{ }^{\star} P<0.05\right)$.

Figure 7 depicts a working hypothesis for the functional role of ACE-2 in hyperoxia-induced lung fibrogenesis. See figure legend for details.

\section{DISCUSSION}

Methods of preventing and/or reducing the effect of oxygen injury remain elusive. Identification of molecular mechanisms of oxygen injury and endogenous protective factors
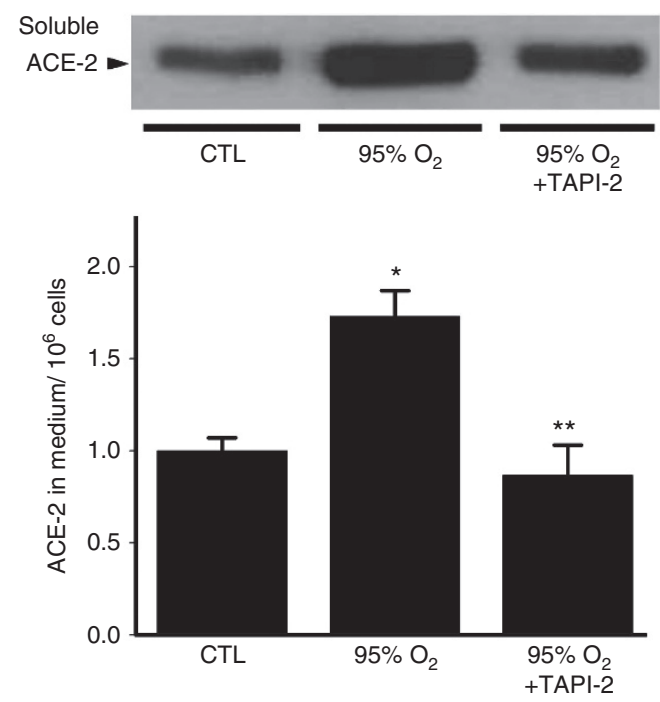

Figure 5. Hyperoxia with normoxic recovery increases soluble angiotensin-converting enzyme-2 (ACE-2) by A TAPI-2-sensitive mechanism. IMR90 cells were exposed to hyperoxic or normoxic gas as described in Figure 1 (with recovery), but in the presence or absence of the ADAM17/ TACE inhibitor TAPI-2. Cell-free culture supernatants were then harvested separately from the cell monolayer and were concentrated and subjected to western blotting for ACE-2 (upper panel). Densitometry (lower panel) demonstrated a significant increase in soluble ACE- 2 in hyperoxia-treated cell supernatants compared to the control $\left({ }^{*} P<0.01 \mathrm{vs}\right.$. CTL by ANOVA and Student-Newman-Keuls multiple comparisons test). Addition of TAPI-2 prevented the increase ${ }^{* *} P<0.01$ vs. $95 \% \mathrm{O}_{2}$ by ANOVA and StudentNewman-Keuls multiple comparisons test). 
might provide much needed targets for future design of interventions. Lang et al (9). recently showed that angiotensin II (ANGII) mediates the profibrotic effect of hyperoxia on cultured human lung fibroblasts, but these authors did not study the potential contributions of ACE-2, which degrades ANGII, to the angiotensin-induced increase in collagen production stimulated by hyperoxia. Moreover, previous work showed that

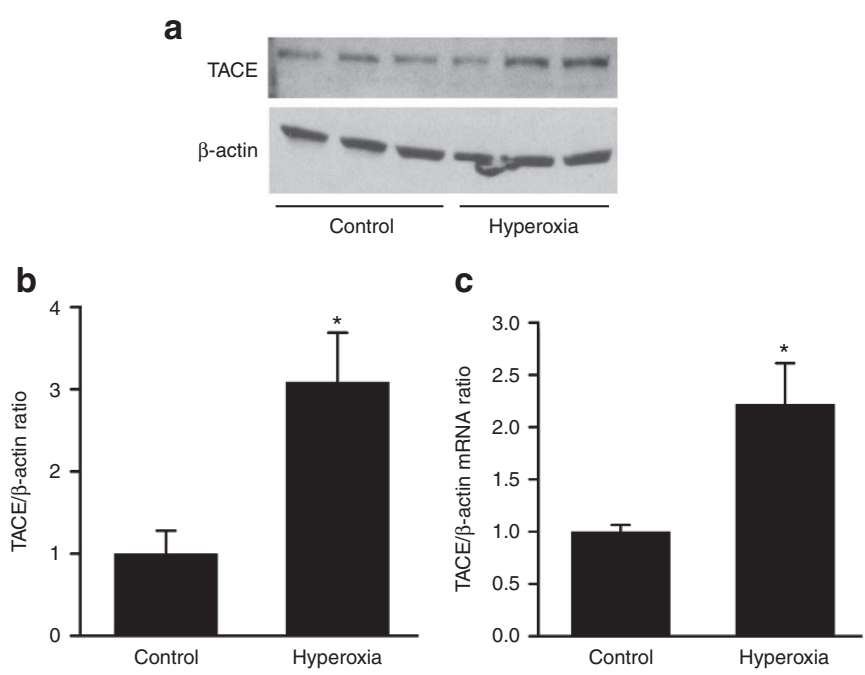

Figure 6. Hyperoxia with normoxic recovery increases ADAM17/TACE in fetal human lung fibroblasts. IMR90 cells were exposed to hyperoxic or normoxic gas as described in Figure 1 (with recovery), then were recovered for western blotting of ADAM17/TACE as described in Materials and Methods. (a) Western blotting for ADAM17/TACE protein was then performed and normalized to $\beta$-actin. (b) By densitometry, hyperoxia with normoxic recovery caused a significant increase in ADAM17/TACE (3.1-fold, ${ }^{*} P<0.05$ by Student's $t$-test). (c) Replicate wells were harvested for recovery of total RNA followed by qRTPCR as described in Methods; hyperoxia with normoxic recovery also caused a significant increase in ADAM17/TACE mRNA (2.2-fold, ${ }^{*} P<0.05$ by Student's $t$-test).
ACE-2, which protects against lung fibrosis in animal models by degrading ANGII, is downregulated in the lungs of patients with idiopathic pulmonary fibrosis through mechanisms yet to be fully identified (11).

Together, these issues led to our hypotheses that: (i) hyperoxia might downregulate ACE-2 and (ii) modulation of ACE-2 might be useful in the management and prevention of hyperoxia-induced lung injury. For example, maintaining high levels of tissue ACE- 2 by prevention of its shedding, and/or replacement of the protein through administration of biologically active enzyme, may hold potential as therapeutic strategies for treating hyperoxic lung injury. In support of this theory, a pharmaceutical preparation of recombinant human ACE-2 (rhACE-2) is currently in clinical trials for acute lung injury (GlaxoSmithKline, pipeline drug GSK2586881). ACE-2, a carboxypeptidase which degrades Angiotensin II, was shown to be protective but downregulated in human interstitial pulmonary fibrosis and in experimentally-induced lung fibrosis by Li et al (11).. A clear role for ACE-2 in modulating acute lung injury was shown by the demonstrations of Imai et al (10). who showed that the absence of ACE-2 in ACE-2 knockout mice exacerbates experimental acute lung injury. To date, the potential role of ACE-2 in hyperoxic lung injury has not been explored.

In recent years, clinicians are seeing less of the classical "old BPD", which is characterized by pulmonary fibrosis alternating with cystic emphysema on chest X-ray with evidence of collagen deposition and fibrosis (19). More recently, the milder form termed "new BPD" has been described, characterized by abnormalities in alveolar septation, pulmonary edema, and less overt fibrosis $(19,20)$. In adult animal models of acute lung injury (10,21), ACE-2 was shown to inhibit lung edema formation and inflammation as well as fibrogenesis. Thus, although little is known about the role of ACE-2 in alveolar septation,

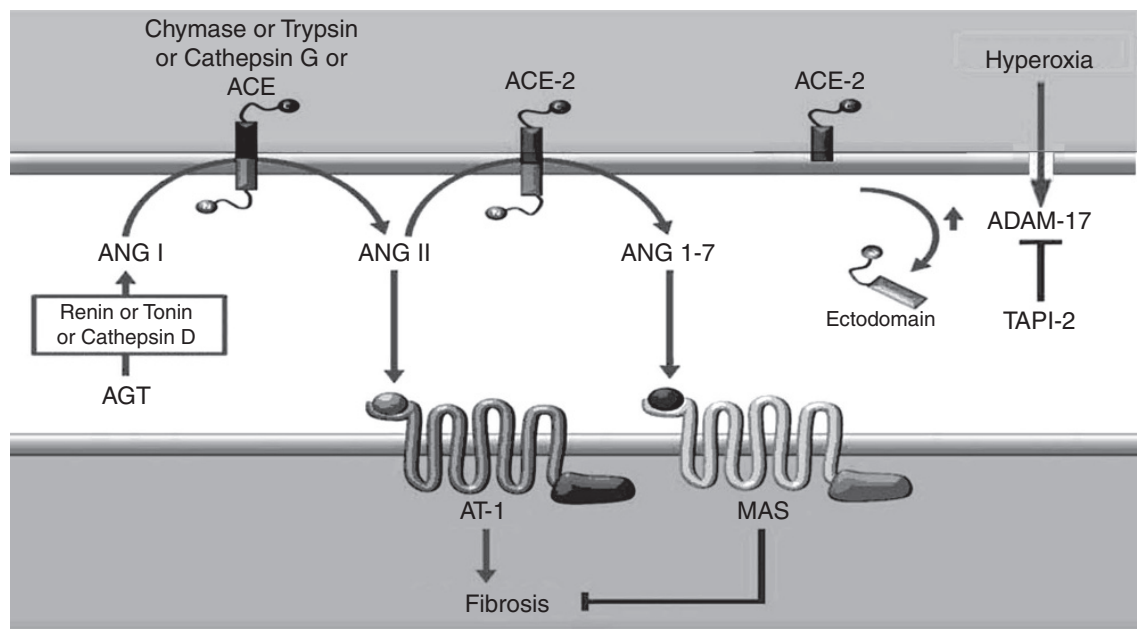

Figure 7. Proposed role of angiotensin-converting enzyme-2 (ACE-2) in hyperoxia-induced lung fibrosis. Angiotensinogen (AGT) produced by either alveolar epithelial cells or myofibroblasts (3) can be cleaved by a variety of pulmonary aspartyl proteases (3) to yield angiotensin (ANG) I, which in turn is cleaved by various lung enzymes to yield the profibrotic ANGII. ANGIl is degraded by ACE-2 to yield the antifibrotic peptide ANG1-7, which acts through its receptor mas. Hyperoxia causes, by mechanisms yet unknown, the release of ACE-2 from the cell through ectodomain cleavage mediated by increased ADAM-17/TACE. Through this mechanism, hyperoxia is theorized to promote fibrosis through both (a) promoting accumulation of ANGII (8,9) and (b) reducing production of the antifibrotic peptide ANG1-7 (21). See text for Discussion. Processes depicted here as intracellular (dark shaded areas) or extracellular (light shading) may well occur in either or both compartments. 


\section{Articles | Oarheet al.}

ACE-2 might be expected to protect against the "new BPD" as well as the more fibrotic "old" form. Regardless, a small percentage of children with BPD today present with classic findings of the "old BPD", and it may be that a continuum exists between the milder forms and the more severe presentations. Therefore, the study of ACE-2 and other components of the RAS in hyperoxic lung injury and the possibility of ameliorating the most severe, and possibly the milder, forms of BPD associated with hyperoxic injury remains critical.

ACE-2 has also been shown to be involved in virus-induced lung disease. ACE-2 is the cellular receptor the SARSCoronavirus, is required for coronaviral entry, and inhibition of its shedding by the TACE inhibitor is related to the severity of the SARS-CoV infection (14-16). Liu et al (22). showed that ACE2 protein levels were significantly downregulated after infection with $\mathrm{H} 1 \mathrm{~N} 1$ viruses, but was dispensable for viral replication. They also found that ACE-2 cleavage could be regulated by influenza neuraminidase. Zou et al (23). showed that in experimental mouse models, infection with highly pathogenic avian influenza A H5N1 virus results in downregulation of ACE-2 expression in the lung and increased serum ANG II levels. Genetic inactivation of ACE-2 causes severe lung injury in $\mathrm{H} 5 \mathrm{~N} 1$-challenged mice, confirming a protective role of ACE-2 in H5N1-induced lung pathologies. The same authors also demonstrated that the administration of recombinant human ACE-2 ameliorates avian influenza H5N1 virusinduced lung injury in mice. Thus, the potential influence of ACE-2 downregulation by hyperoxic gas on viral infection will be an interesting topic for future investigation.

Growing evidence in the study of the role(s) of ACE-2 in normal pulmonary physiology or pulmonary pathophysiology has demonstrated that alveolar epithelial cells are the primary site of ACE-2 expression in mice (24), but relatively less attention has been directed toward understanding the role of fibroblasts in the ACE-2 mediated pulmonary physiology or pulmonary pathophysiology. To our knowledge, the present study is the first to demonstrate that ACE-2 is expressed by human lung fibroblasts and is downregulated by hyperoxia. It is also the first to document that this downregulation could be prevented by TACE antagonist in hyperoxia-treated cells. Given the known function of ACE-2 in preventing lung collagen deposition in experimental lung fibrosis (11), its expression by the fibroblastic cells most responsible for collagen synthesis is not surprising. Indeed, other authors have shown that lung collagen accumulation in neonatal rats exposed to hyperoxic gas could be prevented by blockers of angiotensin receptor AT1 (25).

The well-studied pulmonary toxicity of hyperoxic gas is known to involve apoptotic cell death of alveolar epithelial cells (26), but exposure of the cell line IMR90 to a high oxygen concentration that is often administered to premature neonates $(95 \%$ O2) did not increase a marker of apoptotic cell death in these fetal human lung fibroblasts (caspase 9, Figure 1). Although the 95\% oxygen did cause a very minor increase in necrotic cells $(<1 \%$ see Table 1$)$, it seems unlikely that this small but statistically significant effect would have physiologic significance. The hyperoxic gas did, however, cause a significant decrease in
ACE-2 (Figure 2); whether or not subphysiologic oxygen concentrations reduce ACE- 2 will be an interesting topic for further research. In the light of all published evidence, the downregulation of ACE-2 would be expected to lead to accumulation of ANG II and its downstream profibrotic pathways (see Figure 7). Indeed, hyperoxic gas was previously shown to increase ANGIImediated collagen synthesis by human lung fibroblasts (9), and either inhibition or gene knockdown of ACE-2 increased autocrine ANGII generation by human lung cells in culture (11). Furthermore, the heptapeptide ANG1-7, the product of ACE-2, was shown to be antifibrotic in lung tissue $(21,27)$ and inhibition of ACE-2 reduces its production (see Figure 7). Accordingly, the reduction of cellular ACE-2 induced by hyperoxia would be expected to promote fibrogenesis through both increasing ANGII and simultaneously decreasing ANG1-7.

The mechanism by which hyperoxia decreases ACE-2 appears to be at the protein level, as no significant change in the mRNA occurred and the decrease was prevented by TAPI2, an inhibitor of the ADAM17/TACE enzyme that cleaves the ACE-2 ectodomain in response to SARS Coronavirus (16). As was demonstrated by Haga et al (15)., the present study also showed that the effect of hyperoxia on ACE-2 appears to involve the upregulation of ADAM17/TACE mRNA and protein (see Figure 6), which in turn mediates enzymatic shedding of the ACE-2 ectodomain and its release into the extracellular space. Determination of the signaling mechanism(s) by which hyperoxia upregulates ADAM17/TACE will be an interesting and compelling topic for future investigations.

It is notable that the decrease in ACE-2 occurred only if the period of hyperoxic exposure was followed by normoxic recovery for $24 \mathrm{~h}$ (Figures 2-6). This sequence of redox poise variation is reminiscent of ischemia-reperfusion injury, in which many key signaling pathways are induced not during the ischemic period, but during the return to normoxia (28). Although unknown at present, the mechanisms induced by hyperoxic/ normoxic cycling may be speculated to involve mitochondrial or other redox sensing mechanisms known to be influenced by reactive oxygen or nitrogen species (29). The elucidation of these pathways will also be a very interesting topic for future inquiry.

In summary, this study showed that ACE-2 is expressed not only by lung epithelial cells (24), but also by fetal human lung fibroblasts. ACE-2 protein and enzyme activity, but not mRNA, were decreased by hyperoxia in cultured fetal lung fibroblasts, but ACE-2 protein was increased in the cell culture supernatants. Inhibition of these effects by TAPI- 2 suggests a mechanism mediated by ADAM-17/TACE-induced shedding of ACE-2 from the cell surface. Since it is known that lung injury and fibrosis can be abrogated by ACE-2, the demonstration of a direct effect of hyperoxia on ACE-2 in human lung cells has significant implications for the pathogenesis of hyperoxic lung injury, fibrogenesis and viral infection of the lungs.

\section{METHODS}

\section{Materials}

The peptide $\mathrm{DX}_{600}$, a competitive inhibitor of ACE-2, was purchased from Phoenix Pharmaceuticals, Burlingame, CA. Antibodies against ACE-2 were obtained from Santa Cruz Biotechnology. TAPI-2 was 
also purchased from Calbiochem, SanDiego CA. The fetal lung fibroblast cell line IMR 90 was purchased from ATCC, Manassas, VA. All other materials were of reagent grade.

\section{Cell Culture}

This study was approved by the Institutional Review Board of Michigan State University, East Lansing, MI. The fetal lung fibroblast cell line IMR-90 was subcultured in complete media and harvested at confluence, and then assayed for ACE- 2 by western blot and enzyme activity. Cells were studied at passage 13-15 in all experiments. To demonstrate ACE-2 assay specificity, we measured the level of ACE- 2 in the presence of its competitive inhibitor, DX600 (11). Cell lines at confluence were then exposed to hyperoxia at $95 \%$ oxygen with $5 \% \mathrm{CO}_{2}$ for $72 \mathrm{~h}$, while incubating in $5 \%$ fetal bovine serum. Control cells were exposed to $21 \%$ oxygen with $5 \% \mathrm{CO}_{2}$ for $72 \mathrm{~h}$ with the same media. At the end of the 72 -h culture period, cells were taken out from the incubators and media aspirated out and rinsed once with serum-free media. We also treated some cells with $10 \mu \mathrm{mol} / \mathrm{l}$ of TAPI-2, before incubating in hyperoxia. These were also allowed to be exposed for $72 \mathrm{~h}$ and were treated under the same conditions as the others described above.

Cells were then allowed to recover by incubating in serum-free media for $24 \mathrm{~h}$ in room air. At the end of the recovery period, cells were harvested with $10 \times$ protease inhibitor cocktail and assayed for ACE- 2 by immunoblotting for reactive protein and ACE- 2 enzyme activity.

\section{Cell Viability}

Cell lines cultured to confluence were treated as described above, with control cells at $21 \%$ oxygen and hyperoxia-exposed cells at $95 \%$ oxygen (both at $5 \% \mathrm{CO}_{2}$ ). After $72 \mathrm{~h}$ exposure in $5 \%$ fetal bovine serum, cells were allowed to recover in serum-free media in normoxic gas $\left(21 \% \mathrm{O}_{2}+5 \% \mathrm{CO}_{2}\right)$ for $24 \mathrm{~h}$. Then, cells were treated with Trypan Blue and viewed under the microscope, and blue stained cells as a marker for dead cells were counted. The cells were then treated with propidium iodide for DNA count as a marker for total cells. A total of at least 400 cells per microscopic field were counted over at least three microscope fields per culture well. The counts from each field in a given culture well were then averaged to obtain the data used to calculate the numbers reported as $n=3$ (three culture wells of 1,200 cells each per condition). The total number of necrotic cells as a percentage of total cells was then calculated. To assess for possible apoptosis, caspase 9 was detected by western blotting of cell lysates collected at the end of the 72-h exposure to hyperoxia or at the end of the 24-h recovery in normoxic gas. Blotting methods are described below.

\section{Western blotting}

Cells were harvested with protease inhibitor (Protease Inhibitor Cocktail P840, Sigma, St Louis, MO). Soluble protein lysate (45 $\mu \mathrm{g})$ were loaded and run on $10 \%$ Tris.HCL polyacrylamide gels, separated by SDS-PAGE, in 10× Tris/glycine/SDS buffer (BioRad, Berkeley, CA). Gels were transferred to Immun-blot polyvinylidene fluoride blotting membrane. Blotting membrane was blocked by $5 \%$ nonfat dry milk in $0.1 \%$ Tween 20 in Tris-buffered saline. Western blot analysis of ACE-2 was performed with anti-ACE-2 polyclonal antibody (1:4,000: Abcam Biotechnology, Cambridge, MA). Antibodies for caspase 9 and ADAM17/TACE were obtained from Cell Signaling, Danvers, MA. To ensure equal loading of proteins, membranes were probed with an antibody against $\beta$-actin (Cell Signaling Technology). Bands were visualized by HRP-conjugated goat anti-rabbit secondary antibody (1:10,000 dilution, Santa Cruz Biotechnology, Santa Cruz, CA) using enhanced chemiluminescence detection by standard techniques.

\section{ACE-2 Enzyme Assay}

IMR-90s raised and treated in six-well collagen I-coated plates (BD Biosciences, Bedford, MA) were harvested in ice-cold complete Tris-HCl buffer (Tris-HCl (pH 6.5), 1× Complete Protease Inhibitor Cocktail EDTA-free (Roche, Indianapolis, IN), and lisinopril $(50 \mu \mathrm{g} / \mathrm{l}$; Sigma-Aldrich). Lisinopril was added to the buffer as a means to block the activity of ACE. In a half-area black 96-well microtiter plate, the fluorogenic peptide substrate for ACE-2, MCA-YVADAPK(Dnp)-OH (R\&D Systems, Minneapolis, MN) was added at a final concentration of $10 \mu \mathrm{mol} / \mathrm{l}$ to $30 \mu \mathrm{l}$ cell lysate (in a total volume of $50 \mu \mathrm{l}$ using complete Tris-HCl buffer) on ice. $\mathrm{DX}_{600}$ (at a final concentration of $10 \mu \mathrm{mol} / \mathrm{l}$;
Phoenix Pharmaceuticals, Burlingame, CA), a competitive inhibitor of ACE-2, was also added to half of the wells to compare enzymatic ACE-2 activity inhibition. The plate was warmed to room temperature and the fluorescence was read on a plate reader $(310 / 20 \mathrm{~nm}$ excitation and 420/50 nm emission) in a BioTek FL600 Microplate Fluorescence Reader (BioTek, Burlington VT) for $30 \mathrm{~min}$. Kinetic readings were normalized to their respective DNA concentrations.

\section{Hoescht Dye DNA Assay}

In a clear 96-well microtiter plate, $4 \mu \mathrm{l}$ of cell lysate and $46 \mu \mathrm{l}$ of complete NP-40 lysis buffer (NP-40, 1× Complete Protease Inhibitor Cocktail ethylenediaminetetraacetic acid-free (Roche)) were added. Total volume was brought up to $100 \mu \mathrm{l}$ with the addition of $50 \mu \mathrm{l}$ Hoescht dye (final concentration of $5 \mu \mathrm{mol} / \mathrm{l}$ ). The microtiter plate was then covered and rocked for $30 \mathrm{~s}$ on medium speed. The plate was then placed in a $37{ }^{\circ} \mathrm{C}$ incubator for $15 \mathrm{~min}$. Fluorescence was read on a plate reader (360/40 $\mathrm{nm}$ excitation and 460/40 emission) (FL600 Microplate Fluorescence Reader).

\section{RNA Isolation and Real-Time PCR}

IMR 90 cells after treatment were extracted for total RNA by TRIzol reagent (Invitrogen, Carlsbad, CA) following the manufacturer's protocol. First-strand cDNA was synthesized from total RNA using Superscript II reverse transcriptase and oligo(dT) ${ }_{12-18}$ (Invitrogen). Real-time PCR was carried out with cDNA synthesized from $50 \mathrm{ng}$ of total RNA, SYBR Green PCR core reagents (Applied Biosystems, Foster City, CA) according to the manufacturer's instructions, and $0.2 \mu \mathrm{mol} / \mathrm{l}$ specific primers for human ACE-2 (sense 5'-CATTGGAGCAAGTGTTGGATCTT-3', and antisense 5'-GAGCTA-ATGCATGCCA-TTCTCA-3'), ADAM17/ TACE (sense 5'-TTGGTGGTAGCAGATCATCG-3' and antisense $5^{\prime}$-CTGGGAGAGCCAACATAAGC- $3^{\prime}$ ) and $\beta$-actin (sense $5^{\prime}$-AGGCCAACCGCG-AGAAGATGACC- $3^{\prime}$ and antisense $5^{\prime}$-GAAGTCCAGGGCGACGTAGC-3'). The thermal profile for ACE- 2 and $\beta$-actin started with $10 \mathrm{~min}$ activation at $95^{\circ} \mathrm{C}$ followed by 40 cycles of denaturation at $95^{\circ} \mathrm{C}$ for $60 \mathrm{~s}$, annealing at $55^{\circ} \mathrm{C}$ for $60 \mathrm{~s}$, extension at $72{ }^{\circ} \mathrm{C}$ for $60 \mathrm{~s}$, and ending with dissociation curve analysis of the PCR products. The thermal profile for ADAM17/ TACE started with $10 \mathrm{~min}$ activation at $95^{\circ} \mathrm{C}$ followed by 40 cycles of denaturation at $95^{\circ} \mathrm{C}$ for $15 \mathrm{~s}$, annealing at $55^{\circ} \mathrm{C}$ for $15 \mathrm{~s}$, extension at $72{ }^{\circ} \mathrm{C}$ for $30 \mathrm{~s}$. Reactions were performed in a StepOnePlus Realtime PCR system instrument (Applied Biosystems). Threshold cycle $\left(C_{\mathrm{t}}\right)$ data were collected using StepOne software version 2.1 (Applied Biosystems). The relative ACE-2 expression was normalized to $\beta$-actin and calculated with the comparative CT method of $2-\Delta \Delta \mathrm{CT}$, where $\Delta \mathrm{CT}=\mathrm{CTACE}-2-\mathrm{CT} \beta$-actin and $\Delta \Delta \mathrm{CT}=\Delta \mathrm{CT}_{\text {treatment }}-\Delta \mathrm{CT}_{\mathrm{co}}$

\section{Cell Culture Media Handling}

Media from cells plated in a total volume of $1 \mathrm{ml}$ were collected in a $15 \mathrm{ml}$ conical tube containing $300 \mu \mathrm{l}$ of $10 \times$ Complete Protease Inhibitor EDTA-free cocktail (Roche). Cell debris from the sample was removed through centrifugation. Samples were prepped for dialysis using Spectra/Por Biotech Cellulose Ester Dialysis Membranes with a MWCO $10 \mathrm{kDa}$ (Spectrum Laboratories, Rancho Dominguez, CA) according to manufacturer's protocol. Membranes containing the samples were then kept at $4{ }^{\circ} \mathrm{C}$ in a $2 \mathrm{l}$ beaker of deionized water with gentle stirring until the media sample became clear $(\sim 2 \mathrm{~d})$. At the completion of dialysis, samples were transferred to a new $15 \mathrm{ml}$ canonical tube and frozen at $-80^{\circ} \mathrm{C}$. Completely frozen samples were lyophilized using the Labconco Freeze Dryer/Freezone 4.5 system according the manufacturer's protocol. Lyophilized samples were diluted in $100 \mu \mathrm{l}$ of sterile deionized water. Western blot for ACE-2 and $\beta$-actin was done by standard protocols.

\section{ACKNOWLEDGMENTS}

Portions of the work conducted by some authors (M.D., H.N., and I.G.) were conducted in partial fulfillment of the requirements for the degree Doctor of Philosophy from Michigan State University.

\section{STATEMENT OF FINANCIAL SUPPORT}

This work was supported by HL-45136 from US National Institutes of Health, Bethesda, MD to B.D.U. and by a grant from the Fellowship Research Fund of Sparrow Hospital, Lansing, MI (to C.I.O.). 


\section{REFERENCES}

1. Lemons JA, Bauer CR, Oh W, et al. Very low birth weight outcomes of the National Institute of Child health and human development neonatal research network, January 1995 through December 1996. NICHD Neonatal Research Network. Pediatrics 2001;107:E1.

2. Ratner V, Slinko S, Utkina-Sosunova I, Starkov A, Polin RA, Ten VS. Hypoxic stress exacerbates hyperoxia-induced lung injury in a neonatal mouse model of bronchopulmonary dysplasia. Neonatology 2009;95:299-305.

3. Uhal BD, Kim JK, Li X, Molina-Molina M. Angiotensin-TGF-beta 1 crosstalk in human idiopathic pulmonary fibrosis: autocrine mechanisms in myofibroblasts and macrophages. Curr Pharm Des 2007;13:1247-56.

4. Weber KT, Sun Y. Recruitable ACE and tissue repair in the infarcted heart. J Renin Angiotensin Aldosterone Syst 2000;1:295-303.

5. Yoshiji H, Kuriyama S, Yoshii J, et al. Angiotensin-II type 1 receptor interaction is a major regulator for liver fibrosis development in rats. Hepatology 2001;34(4 Pt 1):745-50.

6. Wang R, Alam G, Zagariya A, et al. Apoptosis of lung epithelial cells in response to TNF-alpha requires angiotensin II generation de novo. J Cell Physiol 2000;185:253-9.

7. Wang R, Ibarra-Sunga O, Verlinski L, Pick R, Uhal BD. Abrogation of bleomycin-induced epithelial apoptosis and lung fibrosis by captopril or by a caspase inhibitor. Am J Physiol Lung Cell Mol Physiol 2000;279:L143-51.

8. Marshall RP, Gohlke P, Chambers RC, et al. Angiotensin II and the fibroproliferative response to acute lung injury. Am J Physiol Lung Cell Mol Physiol 2004;286:L156-64.

9. Lang YD, Hung CL, Wu TY, Wang LF, Chen CM. The renin-angiotensin system mediates hyperoxia-induced collagen production in human lung fibroblasts. Free Radic Biol Med 2010;49:88-95.

10. Imai Y, Kuba K, Rao S, et al. Angiotensin-converting enzyme 2 protects from severe acute lung failure. Nature 2005;436:112-6.

11. Li X, Molina-Molina M, Abdul-Hafez A, Uhal V, Xaubet A, Uhal BD. Angiotensin converting enzyme-2 is protective but downregulated in human and experimental lung fibrosis. Am J Physiol Lung Cell Mol Physiol 2008;295:L178-85.

12. Jiang JS, Lang YD, Chou HC, et al. Activation of the renin-angiotensin system in hyperoxia-induced lung fibrosis in neonatal rats. Neonatology 2012;101:47-54.

13. Chou HC, Lang YD, Wang LF, Wu TY, Hsieh YF, Chen CM. Angiotensin II type 1 receptor antagonist attenuates lung fibrosis in hyperoxia-exposed newborn rats. J Pharmacol Exp Ther 2012;340:169-75.

14. Li W, Moore MJ, Vasilieva N, et al. Angiotensin-converting enzyme 2 is a functional receptor for the SARS coronavirus. Nature 2003;426:450-4.

15. Haga S, Nagata N, Okamura T, et al. TACE antagonists blocking ACE2 shedding caused by the spike protein of SARS-CoV are candidate antiviral compounds. Antiviral Res 2010;85:551-5.
16. Haga S, Yamamoto N, Nakai-Murakami C, et al. Modulation of TNFalpha-converting enzyme by the spike protein of SARS-CoV and ACE2 induces TNF-alpha production and facilitates viral entry. Proc Natl Acad Sci USA 2008;105:7809-14.

17. Black RA, Rauch CT, Kozlosky CJ, et al. A metalloproteinase disintegrin that releases tumour-necrosis factor-alpha from cells. Nature 1997;385:729-33.

18. Lambert DW, Yarski M, Warner FJ, et al. Tumor necrosis factor-alpha convertase (ADAM17) mediates regulated ectodomain shedding of the severe-acute respiratory syndrome-coronavirus (SARS-CoV) receptor, angiotensin-converting enzyme-2 (ACE2). J Biol Chem 2005;280:30113-9.

19. Mosca F, Colnaghi M, Fumagalli M. BPD: old and new problems. J Matern Fetal Neonatal Med 2011;1:k80-2.

20. Vento G, Capoluongo E, Matassa PG, et al. Serum levels of seven cytokines in premature ventilated newborns: correlations with old and new forms of bronchopulmonary dysplasia. Intensive Care Med 2006;32:723-30.

21. Shenoy V, Ferreira AJ, Qi Y, et al. The angiotensin-converting enzyme 2/ angiogenesis-(1-7)/Mas axis confers cardiopulmonary protection against lung fibrosis and pulmonary hypertension. Am J Respir Crit Care Med 2010;182:1065-72.

22. Liu X, Yang N, Tang J, et al. Downregulation of angiotensin-converting enzyme 2 by the neuraminidase protein of influenza A (H1N1) virus. Virus Res 2014;185:64-71.

23. Zou Z, Yan Y, Shu Y, et al. Angiotensin-converting enzyme 2 protects from lethal avian influenza A H5N1 infections. Nat Commun 2014;5:3594.

24. Wiener RS, Cao YX, Hinds A, Ramirez MI, Williams MC. Angiotensin converting enzyme 2 is primarily epithelial and is developmentally regulated in the mouse lung. J Cell Biochem 2007;101:1278-91.

25. Chou HC, Lang YD, Wang LF, Wu TY, Hsieh YF, Chen CM. Angiotensin II type 1 receptor antagonist attenuates lung fibrosis in hyperoxia-exposed newborn rats. J Pharmacol Exp Ther 2012;340:169-75.

26. Wang J, Liu W, Peng Y, et al. Synergetic effect of $\alpha$-lipoic acid with keratinocyte growth factor on protecting alveolar epithelial type II cells of rat fetus from hyperoxia - induced injury. Cell Physiol Biochem 2014;33: 953-66.

27. Uhal BD, Li X, Xue A, Gao X, Abdul-Hafez A. Regulation of alveolar epithelial cell survival by the ACE-2/angiotensin 1-7/Mas axis. Am J Physiol Lung Cell Mol Physiol 2011;301:L269-74.

28. Kulkarni AC, Kuppusamy P, Parinandi N. Oxygen, the lead actor in the pathophysiologic drama: enactment of the trinity of normoxia, hypoxia, and hyperoxia in disease and therapy. Antioxid Redox Signal 2007;9: 1717-30.

29. Agarwal B, Stowe DF, Dash RK, Bosnjak ZJ, Camara AK. Mitochondrial targets for volatile anesthetics against cardiac ischemia-reperfusion injury. Front Physiol 2014;5:341. 